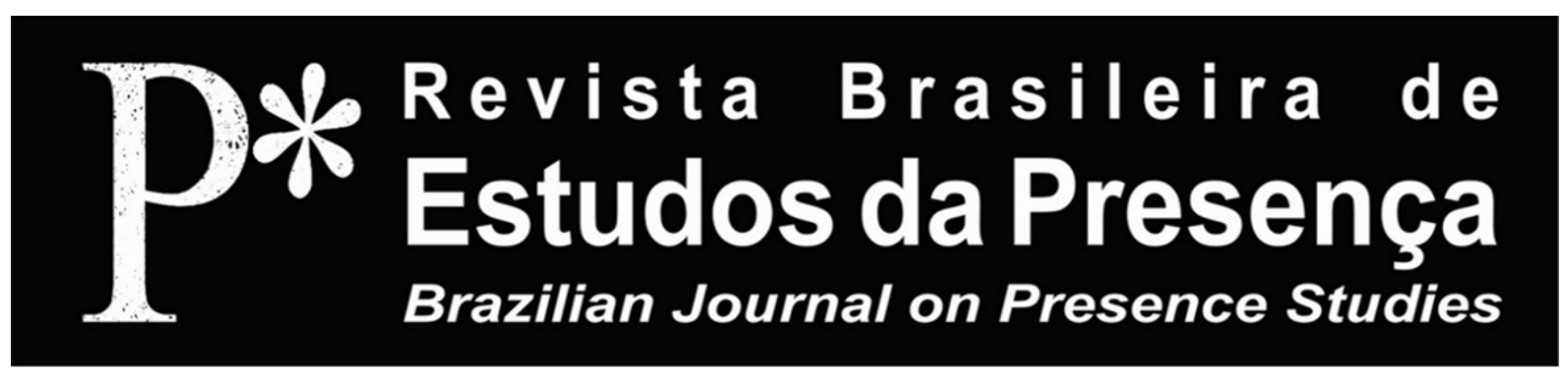

E-ISSN 2237-2660

\title{
4D Art: corps réels et virtuels, une réalité augmentée
}

\author{
Michel Lemieux \\ Victor Pilon \\ Compagnie Multidisciplinaire 4D Art - Montréal, Québec, Canada
}

Marta Isaacsson

Universidade Federal do Rio Grande do Sul - UFRGS, Porto Alegre/RS, Brésil

RÉSUMÉ - 4D Art: corps réels et virtuels, une réalité augmentée - La compagnie canadienne 4D Art surprend le regard du public et interroge les sens de réalité et présence, par l'interaction scénique de corps réels et virtuels en mouvement. Pour comprendre le processus créateur des spectacles de 4D Art, on présente un entretien inédit réalisé avec les directeurs artistiques Michel Lemieux et Victor Pilon. Les motivations artistiques du jeu réel et virtuel, les procédés employés dans la création des figures virtuelles et les défis vécus par les acteurs sont exposés par les créateurs.

Mots-clés: 4D Art. Théâtre. Technologie. Virtuel. Réalité Augmentée.

ABSTRACT - 4D Art: real and virtual bodies, an augmented reality - The Canadian company 4D Art fascinates the eye of the audience and questions their senses of reality and presence through the interaction on stage of the real and virtual bodies' movements. To understand the creative process of 4D Art's multimedia scene, we present an exclusive interview with the artistic directors Michel Lemieux and Victor Pilon. The artistic motivations of the real and virtual game, the procedures employed in the creation of virtual figures and the challenges faced by the actors are exposed by the creators.

Keywords: 4D Art. Theatre. Technology. Virtual. Augmented Reality.

RESUMO - 4D Art: corpos reais e virtuais, uma realidade aumentada - A companhia canadense 4D Art fascina o olhar do público e interroga seus sentidos de realidade e presença pela interaçáo cênica dos movimentos de corpos reais e virtuais. Para compreender os processos de criação da cena multimídia de 4D Art, apresenta-se uma entrevista exclusiva realizada com os diretores artísticos Michel Lemieux e Victor Pilon. As motivaçóes artísticas do jogo real e virtual, os procedimentos empregados na criação das figuras virtuais e os desafios enfrentados pelos atores aparecem nas palavras dos criadores.

Palavras-chave: 4D Art. Teatro. Tecnologia. Virtual. Realidade Aumentada. 
Dans la cartographie de la production contemporaine des arts du spectacle (théâtre, danse, performance), le Québec apparaît comme une région privilégiée en expérimentations artistiques à partir de technologies d'images. Quand on pense à cette région du Canada et à la scène marquée par la technologie, le nom du metteur en scène, acteur et cinéaste Robert Lepage vient immédiatement à l'esprit. Toutefois, depuis les années 1980, d'autres artistes de cette région d'Amérique du Nord développent aussi d'importantes recherches artistiques grâce à la technologie de l'image. Dans ce sens, on peut mentionner les compagnies Ubu Compagnie Création, Les Deux Mondes, Arbo Cyber, théâtre, Kondition Pluriel, Carbone 14, les artistes en résidence à Recto-Verso (espace expérimental pluridisciplinaire géré par la Ville de Québec) et 4D Art. Ce dernier collectif, fondé en 1983, se trouve sous la direction artistique de Michel Lemieux et de Victor Pilon, qui ont accepté d'accorder cet entretien exclusif à la Revista Brasileira Estudos da Presença.

La formation multidisciplinaire de ces deux artistes - théâtre, musique, arts visuels - permet au 4D Art de réaliser des performances artistiques sur différents fronts, des œuvres créées autant pour le théâtre que pour les musées et le cirque. Elle explique également le principe de non-hiérarchie adopté dans l'articulation des différents éléments expressifs afin de composer une création artistique qui mobilise tous les sens du spectateur. Parmi les créations scéniques de ces dernières années, on peut rappeler Anima (2002), La Tempête (2005), Norman (2007), La Belle et la Bête (2011) et Icare (2012) ${ }^{1}$. Les spectacles de Lemieux et Pilon ont dépassé les frontières du Canada pour aller avec succès à la rencontre du public de nombreux pays: les États-Unis, la France, l'Angleterre, l'Autriche, l'Allemagne, l'Espagne, l'Australie, la Colombie, le Venezuela, le Mexique, la Chine, la Corée, la Thaïlande et le Japon. La contribution artistique de Lemieux et Pilon au rayonnement de la culture canadienne est largement reconnue et la médaille de l'Ordre des Arts et des Lettres du Québec a été attribuée aux deux créateurs en 2014.

L'impact des productions scéniques de $4 D$ Art est étroitement lié aux résultats surprenants de l'imagerie, obtenus par la recherche de nouvelles méthodes de création d'image virtuelle pour la scène. Lemieux et Pilon ont notamment renouvelé la technique Pepper's ghost $^{2}$, développée par le scientifique britannique John Henry Pepper, au XIX ${ }^{\mathrm{e}}$ siècle, dans la production de fantasmagories. Grâce à 


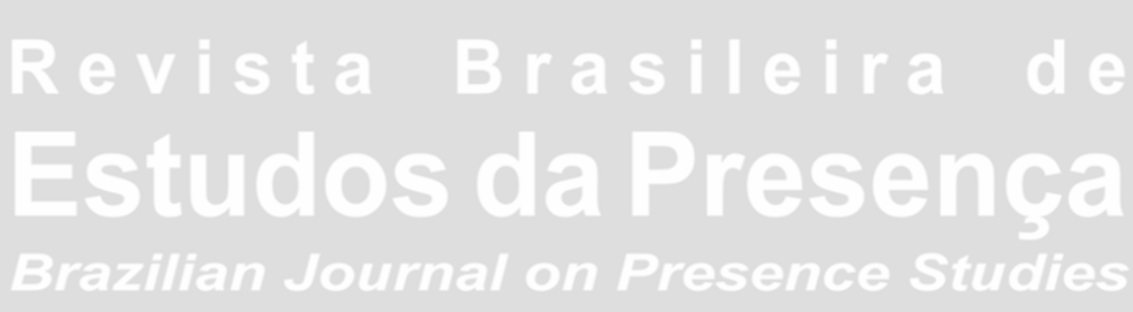

un système de projection sur un support invisible, à un raffinement de l'effet illusoire, les créateurs canadiens surprennent le regard du spectateur, en produisant des images à trois dimensions d'objets, de personnes et d'animaux absents errant sur la scène, comme s'ils étaient effectivement présents. Présentés en entier et sous une forme holographique, les acteurs virtuels, qui semblent en trois dimensions, partagent la scène avec les vrais acteurs. Le spectateur se trouve alors devant l'entrelacement de la présence des corps des acteurs vivants avec les "effets de présence" (Feral; Perrot, 2012) des corps des acteurs absents, dont les images ont été préalablement enregistrées. Face à un jeu d'écoute entre le réel et le virtuel, le public a l'impression de la réalité, de la présence et de l'imaginaire qui sont croisés.

Étant donné que le direct caractérise par nature les arts de la scène, l'utilisation d'images virtuelles au théâtre est souvent controversée. Dans le cas particulier du spectacle La Belle et La Bête (2011) ${ }^{3}$, qui a donné lieu à l'entretien présenté ici, la distinction entre le réel et le virtuel est cependant clairement marquée et articulée à l'intrigue. La figure virtuelle de la sœur de la Belle, par exemple, est toujours représentée dans une taille démesurée par rapport au corps de l'actrice qui joue sur scène le rôle de la protagoniste. Le spectacle distingue le réel et le virtuel et tire profit de cette différence dans la construction narrative en établissant une distinction entre la réalité et la mémoire ou l'imagination des personnages.

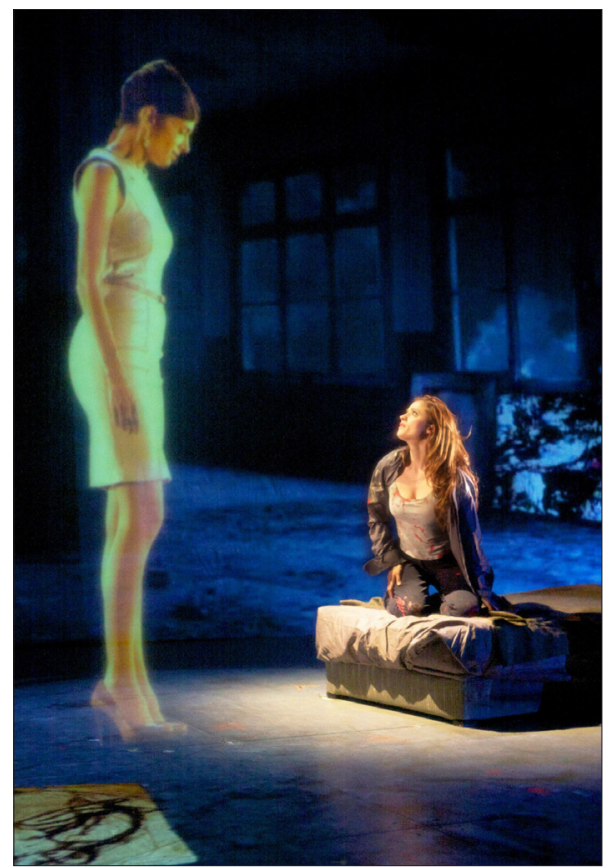

Image 1 - La Belle et la Bête, Paris (2012). Photo: Victor Pilon. 


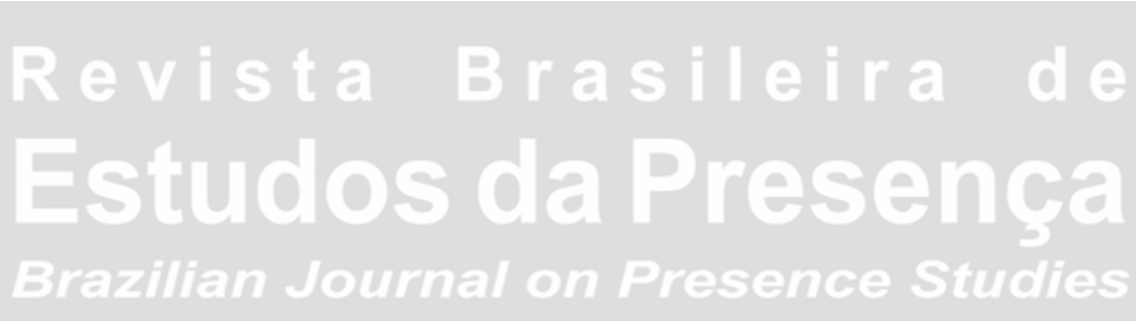

L'intrigue du spectacle se développe sur deux plans à partir des textes de Pierre-Yves Lemieux, inspirés par le conte classique de Madame Villeneuve (1740). Comme dans la vie, il y a le monde que l'on voit et le monde que l'on ne voit pas, celui des désirs, des souvenirs, des peurs et des fantasmes. Ainsi, des figures se joignent aux protagonistes de l'intrigue - la jeune Belle, la Bête et Madame, qui joue dans l'intrigue le rôle du narrateur mais qui, à un moment donné, se transforme en fée. Ces figures représentent l'univers psychique des deux protagonistes. Alors que les acteurs réels incarnent la Belle et la Bête, des personnages virtuels surgissent pour révéler l'univers mental, les pensées, les douleurs et les désirs des protagonistes. Ce qui est impressionnant, c'est que les figures virtuelles ne sont pas statiques et, grâce à un accord millimétré de mouvements, un jeu d'échange entre les personnages réels et virtuels se produit sous le regard du public. Ainsi, la souffrance devant la laideur physique est représentée dans la scène où le personnage de la Bête, incarnée par un véritable acteur, voit apparaître devant lui un corps virtuel qui est son image parfaite du passé. Le conflit intérieur du protagoniste est exprimé alors, dans une lutte acharnée entre l'acteur réel et son personnage virtuel, un jeu finement réglé entre les mouvements en direct de l'acteur et ceux préalablement enregistrés de son image holographique.

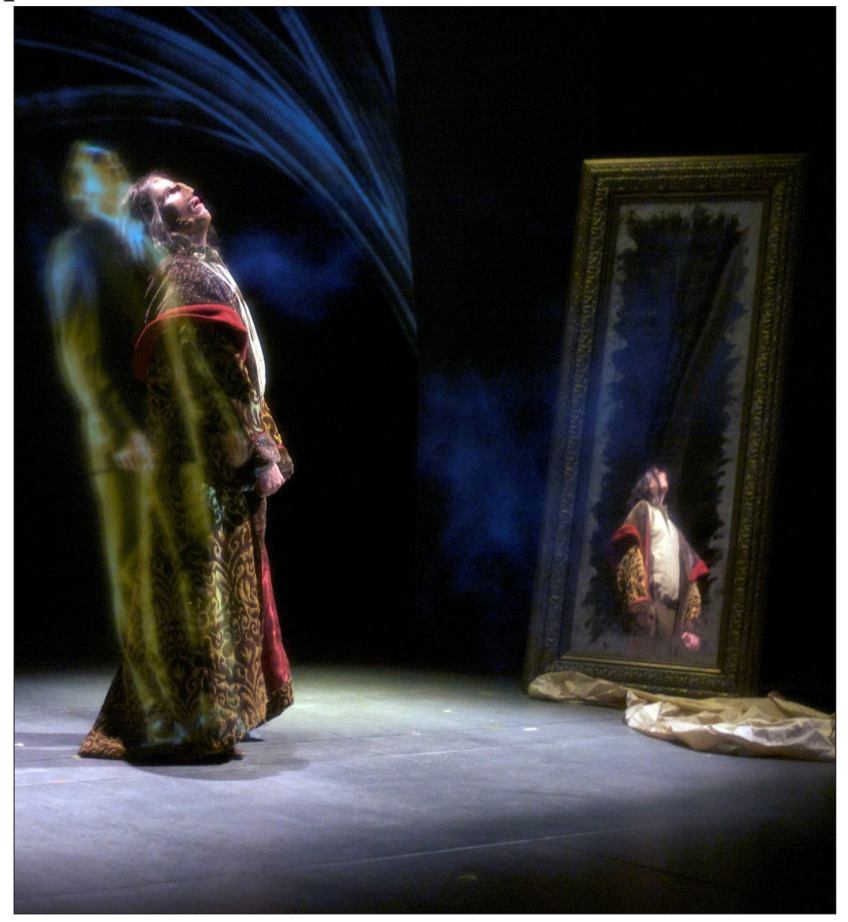

Image 2 - La Belle et la Bête, Paris (2012). Photo: Victor Pilon.

Michel Lemieux; Victor Pilon; Marta Isaacsson - 4D Art: 
L'évolution des tensions internes de la Belle est aussi donnée à voir dans un jeu entre le réel et le virtuel, à travers le dialogue entre la protagoniste incarnée par l'actrice sur scène et l'image virtuelle de sa sœur, dont l'image géante commence à se rétrécir après un moment, si bien que Belle peut recouvrir sa sœur avec un seau.

Il convient de noter que les figures holographiques, même en trois dimensions, ne possèdent pas une texture solide, ce qui permet au spectateur de voir ce qui est derrière eux. Cette apparence immatérielle de la figure virtuelle contribue à renforcer le sentiment d'appartenance à une épaisseur de la réalité qui n'est pas perçue par l'œil. Les images virtuelles de 4D Art séduisent précisément non seulement parce qu'elles semblent réelles mais aussi parce qu'elles comportent une densité irréaliste, qui leur permet d'être la représentation du monde qui ne se voit pas.

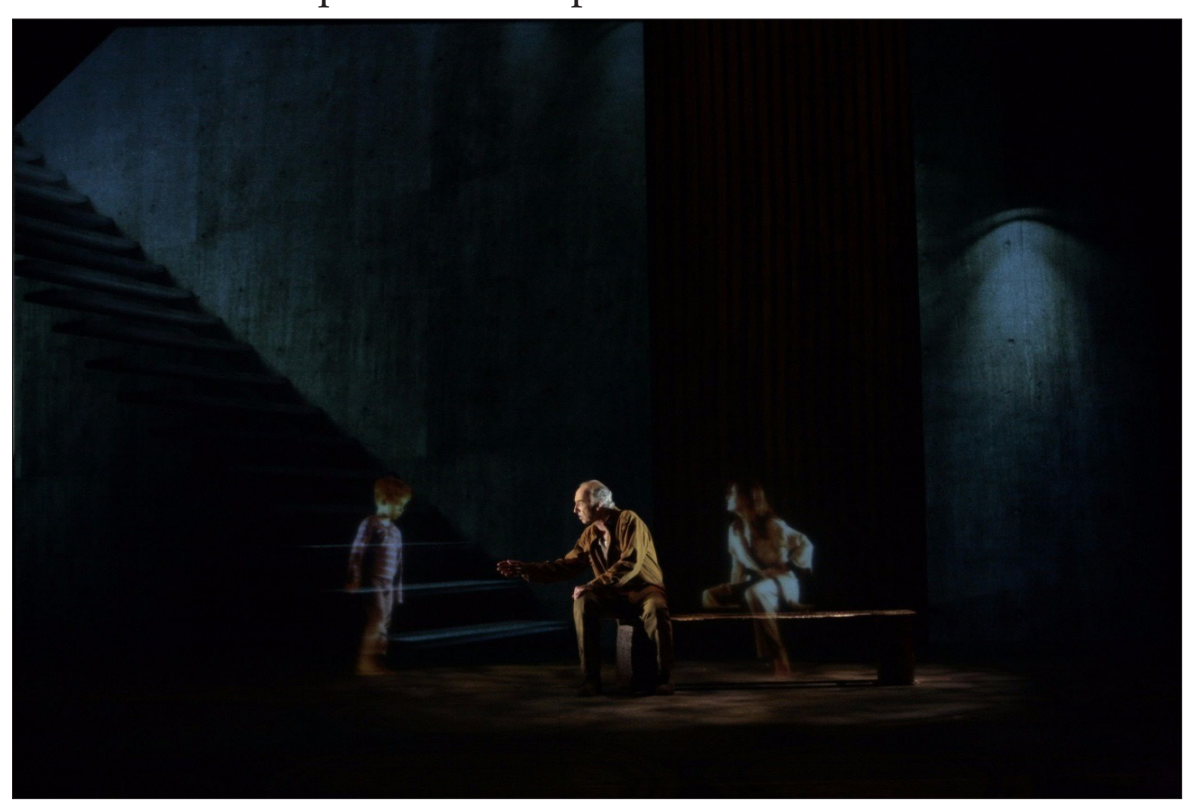

Image 3 - Icare, Compiègne (2015). Photo: Dave Macleod.

En définissant "l'effet de présence", Féral et Perrot (2012, p. 12) rappellent l'installation La Plume et Le Pissenlit d'Edmond Couchot et Michel Bret, en soulignant que l'effet est directement associé à "l'interaction apparente entre l'écran et le spectateur", au moment où les pétales volent vers le bas immédiatement après le souffle de l'utilisateur sur l'écran. Dans le cas de la scène de 4D Art, "l'effet de présence" est également renforcé par une situation d'interactivité. En effet, c'est grâce à l'interaction illusoire entre les acteurs réels et les objets et les acteurs virtuels que "l'effet de présence" de ces der- 
niers se matérialise. Le théâtre dramatique a toujours été fondé sur l'absence des personnages. Dans la scène de 4D Art, les absences se multiplient. Les acteurs sont non seulement appelés à rendre présents les personnages absents qu'ils représentent mais aussi à simuler la présence de leurs partenaires virtuels à travers un ballet subtil de regards et de mouvements.

On peut employer les termes multimédia, interdisciplinaire et même mixmédia pour désigner la scène de Lemieux et Pilon, comme le soulignent certains critiques. Mais pourquoi ne pas l'appeler théâtre? Parce que le spectacle mobilise une diversité de médias? Toutefois, avec des mots, des costumes, de la musique et d'autres éléments, la diversité des médias a toujours fait partie du théâtre. En fait, le théâtre a toujours été un art multimédia. Très tôt, il a accueilli des dispositifs audiovisuels, a accepté le virtuel sur le plateau, comme en témoignent les expériences de Piscator et Meyerhold ${ }^{4}$, au début $\mathrm{du} \mathrm{XX}^{\mathrm{e}}$ siècle.

Il est vrai que, d'un point de vue technique, les performances des 4D Art, ainsi que d'autres créations contemporaines, entretiennent des rapports étroits avec le cinéma et la vidéo. Cependant, lors de la réalisation de l'événement scénique, il n'y a pas de traces réelles du cinéma, même si au cours de la pré-production la technique du filmage correspond au processus de composition des personnages virtuels. En d'autres termes, même si les acteurs virtuels sont composés par le biais de documents filmés préenregistrés, il est difficile de croire que le spectateur a l'impression d'être au cinéma. D'une part, la disparition apparente du support de projection favorise les opérations propres à la réception des arts vivants, différentes de celles du cinéma traditionnel. D'autre part, on ne doit pas oublier que les spectacles de 4D Art incluent la performance en direct des acteurs et/ ou des danseurs devant un public soumis à des variations inhérentes dans les arts vivants. L'éphémérité propre au théâtre se retrouve toujours. Par ailleurs, les spectacles de 4D Art ne correspondent pas à un simple spectacle de sons et d'images. La composition scénique est en effet réalisée en vue de la construction d'un espace-temps différent de celui du spectateur, dans un mouvement propre à la théâtralité. Chez Lemieux et Pilon, le spectateur se déplace mentalement de sa chaise pour participer au drame.

Bien évidemment, les créations de 4D Art ne correspondent pas à la notion de théâtre en tant que travail qui privilégie le texte dra- 
matique ou la conception du théâtre en tant que travail qui privilégie le travail de l'acteur. Ils exploitent sans hiérarchie tous les moyens d'expression. La magie des 4D Art réside même dans l'apparente coexistence, dans le temps et l'espace, du vivant et du virtuel. On considère que la scène de Lemieux et Pilon présente un théâtre de "réalité augmentée" (Azuma, 1997, p. 2), dans lequel les objets virtuels sont insérés en direct, mêlés à de véritables acteurs et objets, dilatant la perception de réalité du spectateur. Devant le spectacle de 4D Art 4, le spectateur est déstabilisé parce qu'il se trouve attiré par une réalité dont il ne maîtrise pas totalement le fonctionnement. Il réprouve le sentiment de la naïveté de l'enfant. La pensée scientifique qui guide sa vie se révèle impuissante et il est séduit par la magie de la création.

Ce théâtre de réalité augmentée ne menace pas l'acteur, car il dépend de son travail, aussi bien dans la production de personnages virtuels que dans le spectacle vivant. Il est vrai cependant qu'il place l'acteur dans un processus créatif singulier, où les compétences de cinéma, théâtre et danse se trouvent toutes nécessaires. Ce théâtre de réalité augmentée exige en fait un acteur "augmenté" par une formation pluridisciplinaire.

Enfin, les performances de 4D Art soulèvent un certain nombre de questions. Comment les effets de la présence d'acteurs virtuels sont-ils produits? Quelles sont les compétences attendues des acteurs dans le jeu scénique avec des personnages virtuels? Quel est le contrat établi avec le spectateur entre la présence et l'effet de présence? Après les nombreuses recherches sur la présence de l'acteur, le potentiel de l'absence qui suscite le regard du spectateur ne mérite-t-il pas d'être aujourd'hui l'objet des discussions des chercheurs de la scène? Sans avoir la prétention d'épuiser toutes les questions que posent les spectacles de $4 \mathrm{D}$ Art, l'entretien ${ }^{5}$ qui suit cherche à enrichir la réflexion sur la relation de la scène contemporaine avec la technologie de l'image. 
Vos créations réunissent en même temps du théatre, de la musique, de la danse, des images virtuelles extraordinaires et des effets de lumière. Vous présentez vos productions artistiques dans les espaces les plus divers, au théâtre, au musée, au cirque, et même au planétarium. Dans ce contexte, vous êtes reconnus comme des artistes multidisciplinaires, des créateurs d'ouvres hybrides. Alors, qu'est-ce que signifie pour vous l'expression multidisciplinarité? Est-ce qu'il s'agit de reprendre le rêve de Wagner de l'aeuvre d'art totalé, auvre de synthèse?

Pilon - Pour nous, l'interdisciplinarité ou la multidisciplinarité existe depuis toujours. Chez les Grecs, on avait déjà le souci d'avoir du mouvement, de la danse, de la musique, du théâtre, du texte. C'est juste la continuité qu'on fait dans notre travail.

Lemieux - Les spécialités se sont développées au cours des siècles. Mais un artiste est un artiste et on peut travailler sur différents médiums. Je pense, par exemple, au cinéma. C'est un art multidisciplinaire. L'opéra aussi est un art multidisciplinaire. On a l'impression que c'est nouveau la multidisciplinarité, mais ça existe depuis toujours.

Pilon - Depuis Aristote...

Lemieux - Ce qui s'est passé, c'est que, quand Aristote est arrivé, le théâtre était plutôt de la littérature. Il y eut cette phrase célèbre: Dehors les bouffons, sortez les gnomes, sortez les bouffons! Le théâtre, c'est la littérature! Mais, avant Aristote, le théâtre était complètement interdisciplinaire et même technologique quelque part. En Grèce, on trouvait du cirque théâtre. Dans les vestiges de théâtre, on a retrouvé des masques en métal où il y avait un porte-voix. C'était très technologique. Puis, imaginez qu'ils travaillaient avec le feu. En effet, pour nous, il y a quelque chose de naturel à transcender les frontières des différentes disciplines, l'expression est totale. Qui dit que dans un spectacle de théâtre, il ne peut y avoir de la danse, ou bien qu'un spectacle de danse ne peut être théâtral? Ce sont, en fait, d'anciennes façons de voir les disciplines. Je pense qu'aujourd'hui on a le goût de travailler en tant qu'artiste. On n'est pas au-delà de la spécialité, puis on aime travailler avec d'autres artistes. Et, ensemble, avec nos talents et nos expériences diverses, on essaye de créer quelque chose qui va apparaître au public. C'est aussi simple que ça. Je pense que le public ne se pose pas les mêmes questions sur les disciplines que 
les critiques. Il est devant une expression qui peut prendre différents chemins, différentes formes.

Pilon - On cherche à solliciter les gens, les spectateurs, autant par le visuel que par la musique... À solliciter le sens. C'est à travers le sens qu'on réussit à émouvoir, à toucher, à provoquer une réflexion. Pour nous, c'est d'expérimenter une autre façon de mise en scène ou de proposition scénographique. Ce n'est pas tout à fait du cinéma, ce n'est pas tout à fait du théâtre. Mais c'est brouiller les frontières entre ces disciplines, pour créer une nouvelle expérience pour le spectateur à la scène.

Lemieux - Oui, je pense que, de toute façon, tous les sujets qu'on peut emprunter, toutes les émotions qu'on peut susciter dans le spectacle reviennent au même. Tout a déjà été dit autant pour le théâtre grec que pour Shakespeare. Tout a été dit. Maintenant, la question est de savoir comment on va redire ça avec des mots d'aujourd'hui, avec une façon de faire qui va rejoindre le public d'aujourd'hui.

Pilon - Avec les outils d'aujourd'hui.

Lemieux - Oui, absolument. On est maintenant entouré de technologie. À ce moment, on dit qu'on en a assez de la technologie. Il y en a donc qui disent qu'on ne devrait pas utiliser la technologie sur scène. Moi, je pense qu'il est naturel d'utiliser la technologie. Quand on a monté $L a$ Tempête ${ }^{7}$, on a beaucoup lu sur la vie de Shakespeare, sur le moment avant l'écriture de la pièce. Il y a des auteurs qui affirment que Shakespeare avait entendu dire que, sur le continent, probablement en France, on faisait du théâtre à l'intérieur. Ce qui, pour Shakespeare, était nouveau, parce que le Globe Theatre était ouvert à l'extérieur et que le théâtre se faisait durant la journée. Alors, il a entendu dire qu'on faisait du théâtre à l'intérieur. Donc qu'on pouvait partir du noir et, avec une lanterne rapprochée de l'acteur, faire apparaître le personnage. Et si l'on partait avec la lanterne, le personnage disparaissait. Maintenant, le faire c'est tout bête, parce que tous les théâtres sont plongés dans le noir. Pour nous, c'est normal de commencer notre canevas théâtral dans le noir. Mais, dans le cas de Shakespeare, c'était quelque chose de complètement nouveau. Alors, semble-t-il, il a écrit La Tempête en pensant à cette nouvelle technologie. Quand on a monté La Tempête, qu'on monte tous nos spectacles, c'est la même lanterne, c'est le même désir de faire de la 
magie, de transcender le sens et d'amener le public dans une réalité différente de la nôtre. Sauf que notre lanterne est devenue plus technologique, mais c'est le même désir.

Vous employez différents germes de départ pour vos créations, la poésie, un film, une fable... Mais la technologie est toujours présente. Dans le choix de la technologie visuelle, quelles sont vos principales attentes visà-vis du public?

Pilon - Je ne sais pas si l'on a des attentes par rapport au public. On a des attentes par rapport à notre création. Premièrement, on veut employer ces outils pour toucher, pour renouveler, comme on disait auparavant. Mais c'est aussi pour surprendre, pour questionner surtout, parce que l'intégration de la projection virtuelle sur scène, avec de vrais avatars, pose tellement de questions sur la réalité, sur l'homme qui se virtualise de plus en plus dans la technologie que nous avons aujourd'hui. Je pense que ça sert à cela. Quel est notre rapport avec le corps, quel est notre rapport avec les autres?

Lemieux - Et avec la réalité? En effet, au-delà du sujet du spectacle, il y a un autre surtexte, au fond, un questionnement de la réalité. Qu'est-ce que c'est la réalité? Qu'est-ce que c'est l'humain? Qu'est-ce que c'est la mort et qu'est-ce que c'est le vivant? Qu'est-ce que c'est le virtuel et qu'est-ce que c'est le réel? Mais aussi, qu'est-ce que c'est l'ordre du rêve? C'est pourquoi j'ai l'impression qu'on ne choisit pas le germe, comme vous dites, sur lequel on base notre création. En fait, j'ai l'impression que ce sont ces sujets-là qui nous choisissent, presque. Ce sont des sujets qui se prêtent à un traitement non réaliste, à un traitement surréaliste. Parce que c'est sûr que le type de travail qu'on fait joue au-delà des mots, joue sur la perception. En fait, ce qu'on demande au spectateur, c'est de s'abandonner, d'être ouvert, de ne pas rester dans sa tête. Il y a une partie de l'œuvre qui passe par le mot, par l'intellect, par l'intelligence, mais il y a une autre partie qui passe par le sensoriel. C'est au-delà des mots, c'est difficile à expliquer. Souvent les spectateurs ont du mal à mettre en mots ce qu'ils ont vu. Certains nous disent qu'à la fin, au moment où le public s'est mis à applaudir, c'était comme un réveille-matin, parce qu'ils avaient l'impression qu'ils rêvaient tout ce temps-là.

Pilon- On joue beaucoup sur l'émerveillement. Le spectateur est devant quelque chose qu'il ne comprend pas nécessairement. Sur- 
tout, l'utilisation de la projection virtuelle sur scène. Quand il ne comprend pas comment cela se fait, il devient comme un enfant, il est émerveillé par cette proposition, il y a quelque chose de magique, d'illusion. Alors, il y a quelque chose qui se déclenche dans le cerveau, je crois, dans les émotions.

Lemieux - Malgré mes cheveux blancs, malgré l'âge adulte, une porte s'ouvre et je deviens disponible pour prendre ce qui est entre les lignes, entre les mots et même entre les images. Je me rappelle la poésie, de la bonne poésie, parce que quand on lit un très bon poème, ça devient une expérience personnelle, parce qu'on se projette dans le poème. Les mots parlent de toi.

Pilon - Les Anglais parlent beaucoup de l'expérience théâtrale qui disbelieves, parce qu'il se passe quelque chose qui nous échappe. Ça ouvre l'esprit.

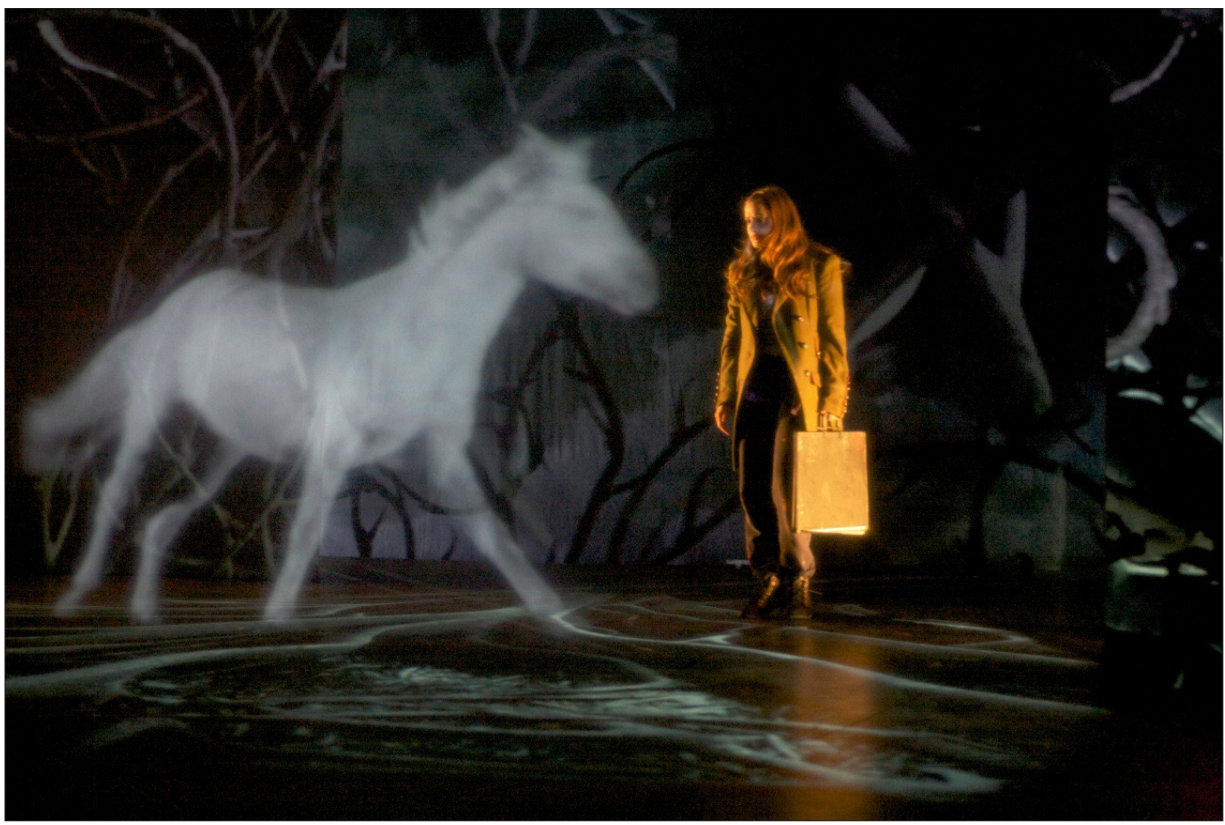

Image 4 - La Belle et la Bête, Paris (2012). Photo: Victor Pilon.

Vous venez d'associer l'emploi de la technologie à l'intérêt de composer un univers surréaliste. Dans La Belle et la Bête, toutefois, on débute sur le réalisme, puis il y a un glissement vers le surréalisme. Etes-vous d'accord? Est-ce que ce mouvement de langage se répète dans d'autres de vos créations?

Lemieux - Oui. On essaye souvent de créer ce mouvement. C'est le principe de la création de la littérature fantastique, même de la 
science-fiction, mais surtout du fantastique. Au départ, il faut inscrire l'histoire dans le réel, auquel le spectateur peut s'identifier. Il ne sort pas complètement aliéné du spectacle, de même que dans toute littérature fantastique. Il y a un moment réaliste et, tout d'un coup, il y a un petit détail qui bascule et à un moment donné il se trouve dans le fantastique. Mais le spectateur n'est pas catapulté dans le fantastique, il est amené, on le prend par la main et on l'amène dans notre univers. On lui dit: 'tu vois, cet univers, il te ressemble, il ressemble à nous tous'. Nous l'amenons, avec son consentement, dans notre labyrinthe, notre voyage onirique, complètement surréaliste.

Le théâtre et la danse tiennent dans la présence des acteurs et des danseurs leur essence. La présence charnelle est comprise comme un élément absolument sacré dans ces arts-là. Vous faites coexister sur scène la présence de corps vivants et de corps virtuels. Quel est, alors, le rôle des acteurs et des danseurs dans cette scène d'image, dans cette réalité hybride que vous composez? Est-ce que l'artiste de cette scène doit avoir des compétences particulières? Par exemple, pour discuter avec un partenaire virtuel?

Lemieux - D’abord, on prend des acteurs extrêmement solides, très forts, qui ont une très grande présence. Parce que quand un acteur est filmé, comme nos acteurs virtuels, il y a quelque chose de la mort. Parfois, on regarde de vieux films et on voit des gens qui sont tous morts. Tous ceux qui jouaient dans ce film-là sont morts. C'est comme regarder un objet qui est rigide. Tandis que l'art vivant, les acteurs sur scène, eux sont tout organes, ils ne sont absolument pas figés. Ils sont dans l'instant présent. Ils sont plus que vivants en fait. L'acteur sur scène représente la vie. Et il doit insuffler un peu de sa vie, de sa présence aux acteurs virtuels, qui ont quelque chose de moins que lui. Parce qu'ils sont morts quelque part. Ils sont filmés, alors, quelque chose s'est arrêté. L'acteur réel doit avoir beaucoup de générosité parce qu'il partage la scène avec quelqu'un qu'il ne voit pas et à qui il doit insuffler une certaine vie. En effet, nos acteurs ne voient pas les personnages virtuels sur scène. Ils les entendent, mais ils ne les voient pas. C'est toute une histoire de mémoire corporelle, la mémoire du corps. Tous les acteurs n'ont pas ça. Les danseurs l'ont, ils l'ont extrêmement, facilement, naturellement. Les danseurs peuvent revenir au même endroit et suivre des repères spatiaux très précis. Certains acteurs sont assez rigides pour y arriver. Parce qu'il a toute une direction du regard, l'acteur réel doit regarder l'acteur 
virtuel dans les yeux, mais il ne le voit pas. L'acteur virtuel bouge, donc, l'acteur réel doit avoir plein de repères au niveau du texte, au niveau du son et au niveau spatial. L'acteur réel doit un peu halluciner le personnage virtuel. Il doit le voir dans sa tête. On sait que les acteurs sont capables de faire beaucoup de choses dans l'abstraction. On dit toujours que les bons acteurs font maintenant du cinéma sur un fond vert, où tout est abstrait. Mais sur une scène, c'est tout une autre chose. Sur une scène drapée de noir, où tout est noir, c'est différent. On pousse un peu plus loin le niveau de l'abstraction. Mais c'est vrai que cela fait partie de la formation de l'acteur d'être capable de se mettre totalement dans une situation qui n'est pas réelle.

Pilon - Mais on doit avouer que, pour les acteurs de théâtre, c'est extrêmement déroutant, surtout au début, de jouer avec l'invisible, d'entendre la voix et de donner la réplique à quelqu'un qu' ils ne voient pas. C'est très dur pour eux. Au début, ils sont perdus, ils cherchent beaucoup. Puis, à un moment donné, ils commencent à halluciner les personnages, à les imaginer là, à jouer avec eux. C'est à ce moment-là que règne la magie. Pour nous, en tant que spectateurs, quand les acteurs croient à ces personnages virtuels, nous commençons aussi à y croire. Mais ça prend un certain temps de maîtriser l'espace, l'environnement.

Mais on a joué quelques pièces où l'acteur virtuel était dans un autre studio et où on avait une caméra qui projetait son image virtuelle en temps réel. On peut alors avoir un échange de répliques, tout ça, qui n'est pas nécessairement emprisonné dans le jeu ou dans l'échange de répliques. Aussi, souvent, c'est l'acteur réel qui va donner le rythme, puisqu'on a le contrôle de l'acteur virtuel et qu'on peut lancer les répliques avec l'acteur virtuel. Il y a une certaine liberté. On s'est aperçu, dans la dernière création d'Icare ${ }^{8}$, que parfois, indépendamment du jeu de l'acteur réel, ça changeait même le sens de l'acteur virtuel. Alors, on a vu les nuances qui peuvent être travaillées et modifiées d'un jour à l'autre, même avec l'acteur virtuel.

Lemieux - Ce qui est difficile pour l'acteur réel sur scène, c'est d'être un peu dans le vide, parce qu'il est sur scène et que les décors sont projetés. Il voit un peu les projections, mais pas beaucoup. Et il ne voit pas le personnage virtuel avec qui il parle. Donc, souvent, l'acteur nous dit: 'J'ai l'impression qu'il ne se passe rien. J'ai l'impression que ce n'est pas intéressant'. Il a l'impression qu'il n'y a que lui sur 


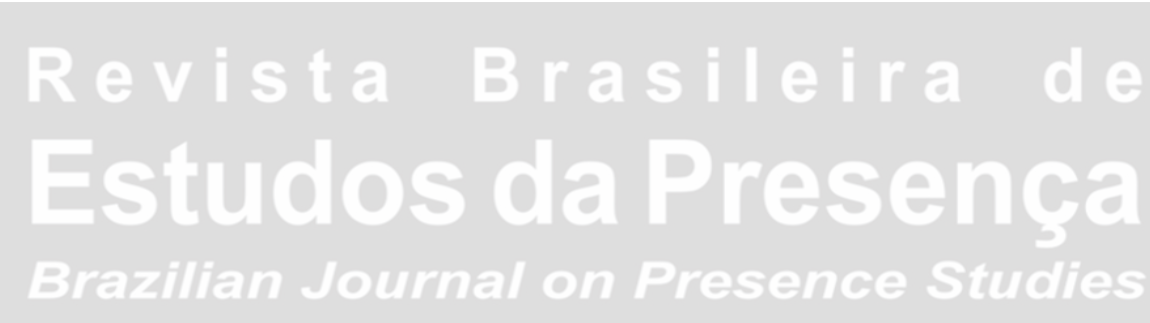

scène. Il dit qu'il a la sensation d'être dans le vide. Alors, quand on montre à l'acteur des séquences vidéo du spectacle, d'habitude, il se trouve surpris. 'Ah, je n'ai pas l'impression d'être dans ce spectacle-là. Je ne vois rien de ce que le spectateur voit'. Là, il comprend et, à ce moment-là, il s'abandonne.

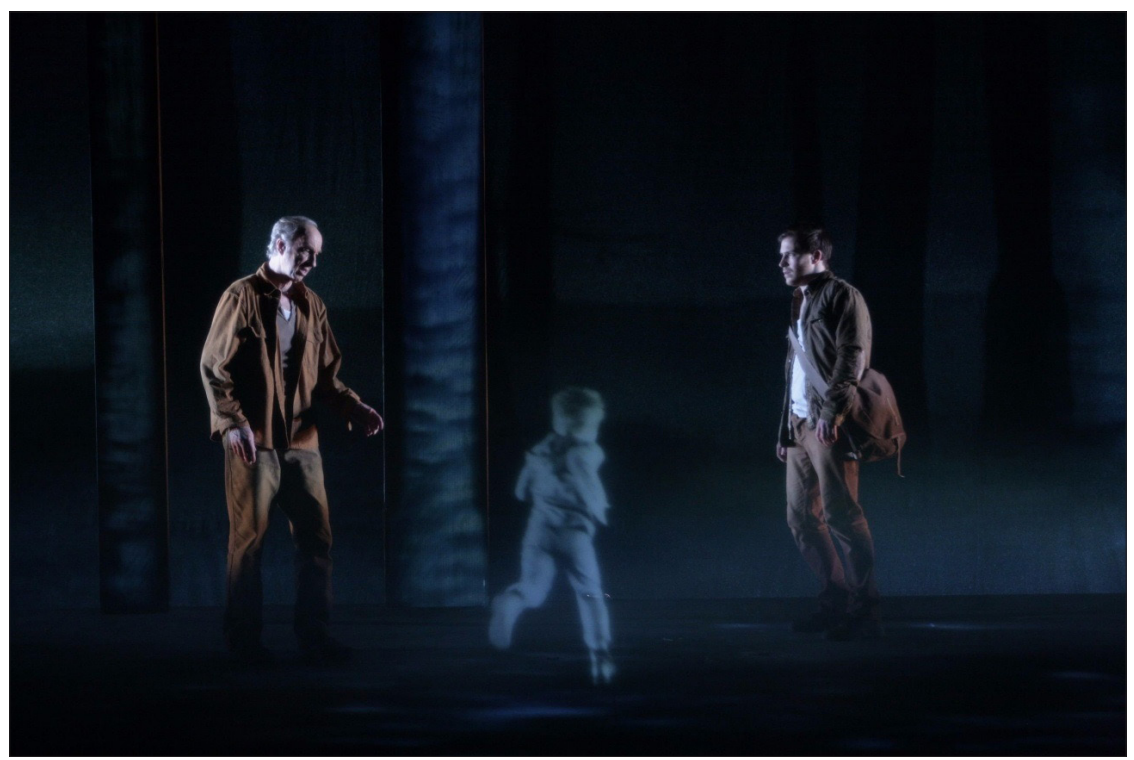

Image 5 - Icare, Compiègne (2015). Photo: Dave Macleod.

Il comprend qu'il a la sensation du vide, mais ce n'est pas ça que le spectateur perçoit. Le spectateur reçoit un plein, parce que l'image est pleine, tout est plein. Quand l'acteur s'abandonne, la magie du théâtre est ouverte et vient nous chercher comme spectateurs dans des zones qui ne sont pas souvent touchées. Parce qu'on a tous connu déjà des gens qui sont décédés, qui sont morts. On se souvient de ces gens-là. C'est la mémoire. Les personnages virtuels peuvent être des personnages de notre mémoire. Ils peuvent aussi être des personnages de notre imaginaire. Donc, à les voir sur scène, on fait une expérience, comme spectateur, souvent, ça va chercher dans des domaines assez intuitifs, qu'on n'arrive pas toujours à rejoindre par un mot.

Toujours sur le processus de création, à quel moment l'acteur ou le danseur commence-t-il à participer? Est-ce qu'au moment où il entre dans le processus de création toutes les questions du décor, des personnages virtuels et les apports techniques sont déjà définis? Ou bien y a-t-il un espace d'échange, d'improvisation entre les acteurs/danseurs et vos idées d'images, les possibilités technologiques, avant la définition de la mise en scène? 
Pilon - D'abord, il y a l'écriture. On travaille, parfois, avec un auteur, un dramaturge. Puis dans l'écriture même du spectacle, on trouve une justification pour intégrer le virtuel. Pourquoi le virtuel? Parce qu'on ne peut pas faire n'importe quelle pièce, monter n'importe quel spectacle. Il doit y avoir une raison réelle d'intégrer cet univers-là. Alors, au début, il y a une écriture qui se fait avant. Après, on réalise le travail de table normal qu'on fait au théâtre, avec des acteurs réels et avec des acteurs virtuels. On répète le texte, on étudie le texte. On approfondit les différentes motivations du jeu, de l'émotion.

Lemieux - Durant toutes les premières semaines de répétition, tous les acteurs sont là, même ceux qui sont virtuels. On fait de la mise en place, on décortique le texte, les intentions, avec les acteurs. Ils sont donc habitués. Au bout de quelques semaines, on passe au tournage. Alors là, l'acteur qui va se retrouver réellement sur scène est habillé en noir. Et l'acteur virtuel va être comme dans un tournage, sauf que c'est un tournage en plans-séquences. Donc, on est d'une certaine manière encore dans le théâtre filmé. Souvent, il s'agit de séquences de 4 ou 5 minutes. Ainsi, il faut que l'acteur connaisse tout son texte, toute la discipline propre au théâtre filmé. Et après, on fait disparaître l'acteur qui sera en scène. Puis on choisit quelle prise on va prendre.

Pilon - Souvent, on fait 5, 10, 20 prises de la même scène pour choisir.

Lemieux - Il s'agit d'un choix éditorial. C'est la direction qui le fait. Après, il y a tout un bloc de répétitions avec l'acteur réel et les acteurs virtuels. Là, il y a une première étape très technique, une mise en place très technique, avec le regard. On met des ' $x$ ' partout sur le sol et l'acteur doit vraiment s'y tenir. Ce que nous disent les acteurs, c'est qu'ils font ça couramment au cinéma. Au cinéma, il y a dix jours de tournage, on leur dit de se déplacer d'un endroit à l'autre, la caméra tourne, c'est très technique. Les acteurs disent que la différence, c'est qu'au cinéma on me demande de réaliser des déplacements très précis dans la journée, mais le lendemain, je ne dois pas les refaire. Une fois le tournage terminé, c'est fini. Vous, vous me demandez de le faire pour chaque séance, pendant $1 \mathrm{~h} 30,2 \mathrm{~h}$. Ce qui veut dire que nos spectacles sont comme des plans-séquences de cinéma qui durent une heure et demie, avec tout ce que ça peut impliquer de technique.

Pilon - Il y a une autre dimension très importante, c'est la voix, c'est le son. On travaille beaucoup avec nos acteurs, puisque au théâtre, 
les acteurs ont tendance à projeter beaucoup la voix pour remplir la salle. Or, nous, on leur pose des micros. Les acteurs réels ont tous des micros...

Lemieux - Ce sont les mêmes micros qu'utilisent les acteurs virtuels et au même endroit, parce que l'idée est d'avoir le même espace sonore.

Pilon - Pour y croire aussi. Il y a alors cette dimension du cinéma. Il faut parfois jouer dans sa tête. On dit aux acteurs: 'Non, il faut jouer plus petit, plus petit, comme au cinéma'. Parce que sinon, on perd cette intimité-là.

Lemieux - Au jeu des micros, on perd quelque chose de naturel, mais, en même temps, on gagne la proximité avec les personnages. Au Théâtre de Chaillot ${ }^{9}$, c'est une grande salle, mais on pouvait même entendre les personnages respirer, comme si on était dans un tout petit théâtre. Quand on filme un acteur, s'il est trop théâtral, s'il joue trop gros, il y a quelque chose qui ne passe pas. Il faut chercher la vérité (donc, les acteurs, même découragés au début, parce que, au Québec, tous les acteurs qui font du théâtre font du cinéma et de la télé. Parce que si on veut gagner sa vie, il faut tout faire). Au départ, lorsqu'on arrive dans un grand théâtre, les acteurs jouent trop grand, ils veulent remplir la salle, ils parlent fort. Mais avec le micro, ce n'est pas nécessaire. C'est déroutant au début pour les acteurs. En fait, ce n'est pas du cinéma, ce n'est pas du théâtre. Il faut trouver un nouveau langage...

\section{La grammaire n'est ni celle du théâtre ni celle du cinéma?}

Lemieux - Oui, on ne peut pas arriver avec la grammaire du cinéma, où on a un plan large, après ça, un plan de deux personnes, après un gros plan... La grammaire du cinéma ne s'applique pas. Quand on fait un personnage virtuel sur scène, on veut qu'il ait une certaine réalité. Il est de plain-pied, souvent il est grandeur nature et il est fait dans un plan-séquence. On veut sa réalité.

Pilon - Parfois, on entre dans l'esprit de l'acteur réel. Alors, on peut fantasmer. On peut faire paraître le personnage plus grand ou plus petit. Changer l'échelle d'un personnage. Ça nous permet de donner à voir ce que nos acteurs pensent.

Lemieux - Il ne s'agit pas de remplacer les acteurs mais de magnifier leur présence. Et ça leur donne l'occasion de travailler avec des acteurs 
qui ne viendraient pas en tournée. Souvent les acteurs qui jouent les personnages virtuels, ce sont de grands acteurs, très connus, qui ne viendraient jamais en tournée parce qu'ils sont trop pris par le cinéma, la télé. Pour nous, nous avons la chance de les avoir dans nos spectacles et, pour eux, ils touchent un droit de présence à chaque représentation.

Après avoir choisi le germe de départ, comment travaillez-vous la conception des images de la scène? Est-ce que vous faites des dessins, des croquis, des maquettes? Puis, est-ce qu'à chaque spectacle, il faut toujours rechercher la bonne technologie, des dispositifs, des équipements plus favorables? Est-ce qu'il y a un certain bricolage des dispositifs, l'emploi d'un équipement avec une fin différente de celle pour laquelle il a été conçu? Est-ce que ça arrive?

Lemieux - Ça arrive tout le temps. Ce qu'on aime, c'est-ce qu'on appelle faire du détournement technologique. On prend des machines, des projecteurs vidéo, des ordinateurs, qui ont certains usages, que beaucoup de gens utilisent, mais nous, on les utilise différemment.

Pilon - On lit le manuel d'instruction à l'envers.

Lemieux - C'est ça. On essaye de transcender. En fait, on ne respecte pas beaucoup la technologie dans le fond. Alors, on brouille les pistes. Et on se prépare, parce qu'il y a à la fois le tournage d'un film et le montage d'un spectacle, alors ça demande beaucoup de préparation. Mais la vie se passe dans la salle de répétitions. L'idée de bien se préparer, c'est être prêt, avoir de bonnes bases pour justement nous permettre d'improviser, nous permettre de créer, de changer les choses qu'on avait prévues. La vie de répétitions fait que ce qu’on fait en répétitions est bien meilleur. Je pense qu'il faut toujours rester ouvert aux accidents qui peuvent être meilleurs que ce qu'on a prévu. Souvent, ce qu'on avait prévu de faire, c'est un tremplin pour aller plus loin avec les acteurs. C'est pourquoi on travaille toujours avec des gens très créatifs, que ce soit des acteurs, des danseurs ou des musiciens. Ce sont des gens qui ne sont pas que des interprètes. Qui peuvent rendre les spectacles humains. Par exemple, dans La Belle et la Bête, on cherchait, on cherchait et on n'arrivait pas à trouver une scène où la Belle se demandait si elle allait venir vers la Bête. On avait un miroir dans le décor, parce que, dans le conte, le miroir est très important. On lui demandait de regarder dans le miroir, mais 
ça ne fonctionnait pas. Un jour, l'actrice a dit: 'Pourquoi je n'ai pas le miroir en face de moi, en virtuel?'. Alors, on a couru sur Internet, on a cherché un cadre de miroir, on a trouvé le cadre et on l'a mis là. Cinq minutes plus tard, il y avait déjà un cadre de miroir virtuel. L'actrice disait: 'Mais vous êtes complètement fous, vous êtes des magiciens, on parle et soudain la chose apparaît'. Donc, c'est sûr qu'il y a des intuitions, comme celle-là, où les intuitions des acteurs, les intuitions des décorateurs, de l'équipe technique sont souvent meilleures que les nôtres.

Pilon - Mais, comme je disais au début, on travaille beaucoup sur le texte, mais on travaille aussi beaucoup sur les maquettes. Souvent, on fait une ébauche de la mise en scène avec la maquette, parce qu'il y a une complexité au niveau de la projection, des déplacements. Il faut donc bien se préparer avant. On travaille alors d'abord sur la maquette. Puis on travaille avec les acteurs, on leur montre la maquette, on leur explique ce qu'on veut faire, dans quel univers ils seront. D'abord, le choix des thèmes ou des histoires qu'on veut raconter, il faut que ce soit des choses qui nous sont très personnelles, des choses qui nous font mûrir, qui nous font en tant qu'artistes, en tant qu'êtres humains. Où ça va nous mener? Est-ce qu'on a quelque chose à dire et qu'est-ce qu'on a à dire? Comment ça va nous transformer, nous, en tant qu'artistes aussi? Ce sont des choses qui doivent avoir une résonance au niveau personnel, ce sont des thèmes qui nous sont chers.

Lemieux - Oui, parce qu'on passe des années à travailler là-dessus. C'est dommage de travailler pendant des années sur quelque chose qui n'est pas vital, important.

Pilon - Il y a la dimension que la technologie est lourde et quand même coûteuse. Elle est chère pour un tas de répétitions. Alors, on doit déroger un peu au standard du théâtre. On ne peut pas mettre en pratique la même façon de travailler du théâtre. Parce qu'au Québec, il y a un tas de semaines de travail à la table, un tas de semaines avec les costumes, un tas de semaines avec le décor. Nous, on essaye d'avoir tous les éléments quand on se met à répéter avec les acteurs, on essaye d'avoir tous les éléments entre les mains pour faire réellement de l'intégration. Sinon, on va faire de la juxtaposition. 

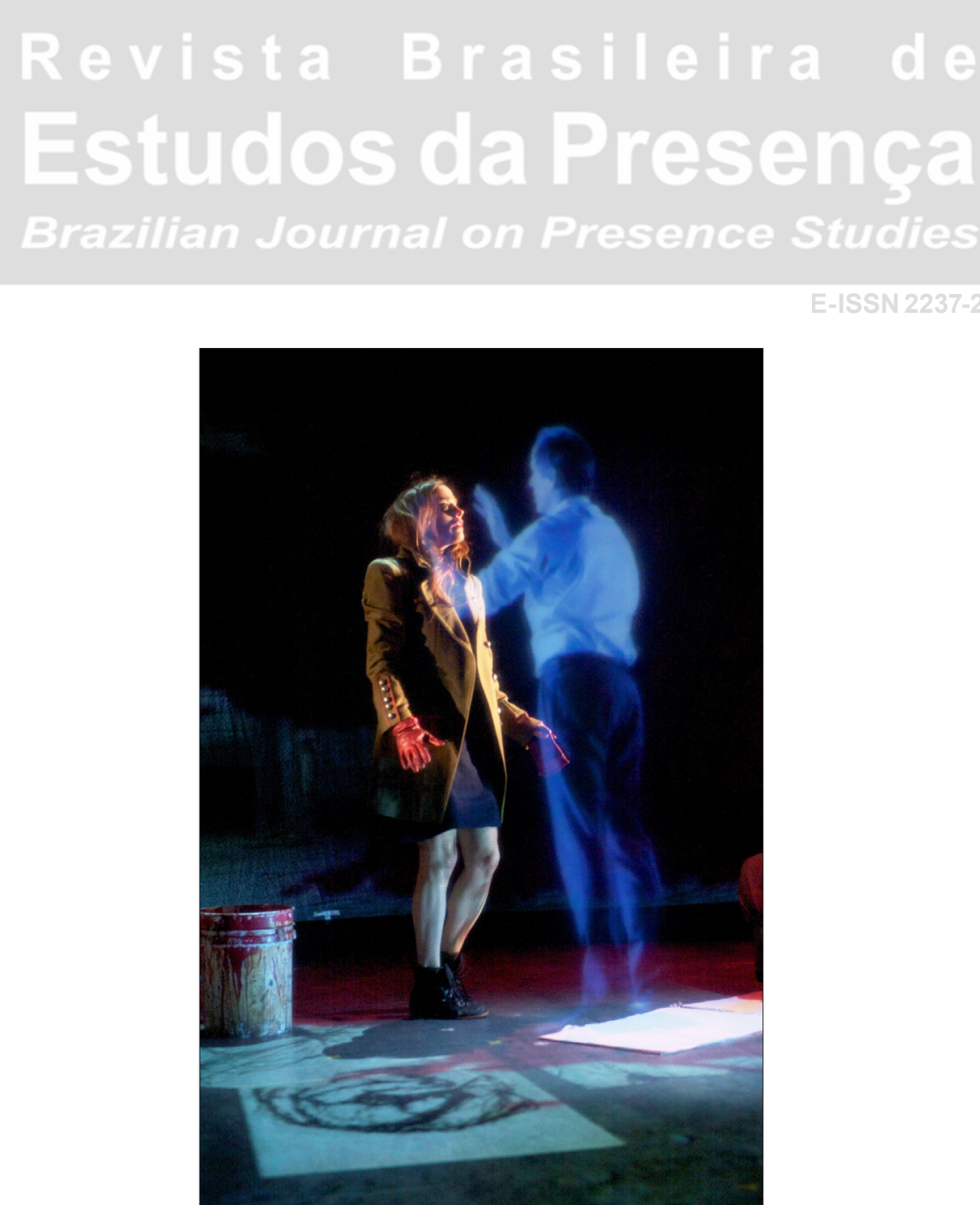

Image 6 - La Belle et la Bête, Paris (2012). Photo: Victor Pilon.

En général, combien de temps employez-vous en répétitions?

Pilon - En général, on doit avoir au moins 4 à 6 semaines de travail avec tous les éléments, avec le virtuel, avec le décor. Ici, au théâtre, on répète seulement une semaine avec tous les éléments, parce que le décor arrive une semaine avant la première. Nous, on répète dans une autre salle séparée, dans un hangar et, dès le départ, on a tous les éléments. C'est seulement de cette façon qu'on a réussi à faire disparaître les frontières et à faire effectivement de l'intégration plutôt que de la juxtaposition. Il y a alors des coûts supplémentaires, puisque toute l'équipe technique doit être présente dès le premier jour. Cela alourdit les frais de production.

Lemieux - Je crois que la multidisciplinarité et l'interdisciplinarité s'appliquent toutes les deux à notre travail. Mais ce que je trouve intéressant dans le mot multidisciplinarité, c'est l'idée de multiplication. Ce n'est pas l'addition d'un spectacle de théâtre avec un spectacle de danse, un spectacle d'arts visuels. C'est plus que de la juxtaposition. Parce que, souvent, on voit de la juxtaposition. On voit des projections et des danseurs, des danseurs devant les projections, mais pas 
de liens entre les deux. C'est la juxtaposition des médiums. Nous, ce qu'on tente de faire, c'est de la multiplication.

Le plus impressionnant dans les compositions visuelles que vous créez, ce sont les projections sans support apparent, en particulier les figures humaines. Alors, pour terminer cet entretien, on voudrait savoir où vous irez après ces expériences d'acteurs virtuels. En ce moment, quelles sont vos recherches technologiques?

Lemieux - Ce qu'on travaille, depuis quelques années, en plus de la virtualité, c'est l'immersion. Au Théâtre de Chaillot, on a fait moins, parce qu'il est très grand. Souvent, on est dans les théâtres à l'italienne qui sont plus fermés, alors, les images sortent vraiment du cadre de la scène et elles vont rejoindre tous les spectateurs dans le parterre. On projette sur les murs, sur le plafond du théâtre, parfois même sur le public. Dans La Belle et la Bête, à Chaillot, l'effet ne fonctionnait pas très bien, parce que l'espace était très large, il n'était pas propice à créer l'immersion. Mais on a joué dans des théâtres historiques avec d'anciens décors qui allaient très bien avec la direction artistique de La Belle et la Bête. Alors, quand on projette les images qui puisent au niveau du théâtre, tout à coup, le spectateur est moins devant quelque chose, mais à l'intérieur, il est vraiment en immersion. C'est ce qu'on veut poursuivre. 


\section{Notes}

${ }^{1}$ Les images des spectacles sont disponibles sur: <http://4dart.com/>.

${ }^{2}$ Effet d'illusion optique: les objets cachés du public semblent présents sur le plateau en fonction de leur reflet sur une vitre inclinée.

${ }^{3}$ Disponible sur: <https://www.youtube.com/watch?v=qExGC7Lpc7g>. La Bête: Vincent Leclerc, François Papineau; La Belle: Bénédicte Décary, Janine Thériault; La Dame: Louise Laprade, Andrée Lachapelle, Diane D’Aquila / Personnages virtuels, La Sœur: Anne-Marie Cadieux, Violette Chauveau; Le Démon: Peter James; Le Cheval: Champagne.

${ }^{4}$ Sur ce sujet: Amiard-Chevrel (1990).

${ }^{5}$ L'entretien a été réalisé via Skype, le 9/01/2015, Porto Alegre-Montréal.

${ }^{6}$ Gesamtkunstwerk ou "l'œuvre d'art totale" est un modèle artistique, inspiré de la tragédie antique grecque, qui prévoit la fusion des arts par l'emploi simultané de nombreux médiums.

${ }^{7}$ Création 2015. Disponible sur: <http://4dart.com/fr/creation/2005/la-tempete/>.

${ }^{8}$ Création 2014. Disponible sur: <http://4dart.com/fr/creation/2014/icare/>. Icare: Julien Lemire; Dédale: Richard Thériault; Le Choryphée: Noëlla Huet / Personnages virtuels: Naucraté: Pascale Bussières; Talos: Maxime Denommée; Icare enfant: Loïk Martineau

9 Théâtre de Chaillot (1937), situé à Paris, est l'un des six théâtres nationaux français. Il comporte trois salles: Jean-Vilar (1250 places); Gémier (420 places) et Maurice-Béjart (80 places).

\section{Références}

AMIARD-CHEVREL, Claudine. Théâtre et Cinéma Années Vingt: une quête de la modernité. Lausanne: L'Agê d'Homme, 1990.

AZUMA, Ronald. A Survey of Augmented Reality. Presence: teleoperators and virtual environments, v. 6, issue 4, p. 355-385, Aug. 1997. Disponible sur: <http://www.cs.unc. edu/-azuma/ARpresence.pdf z. Consulté: 15 Sept. 2015.

FÉRAL, Josette; PERROT, Edwige. De la présence aux effets de présence. In: FÉRAL, Josette. Pratiques Performatives: body remix. Rennes/Montréal: Presse de l'Université du Québec / Presses Universitaires de Rennes, 2012. P. 11- 40. 
Victor Pilon est metteur en scène, scénographe, concepteur visuel et photographe. En 1990 il rencontre Michel Lemieux et fonde le 4D Art dont ils sont toujours concepteurs et codirecteurs artistiques.

E-mail: info@4dart.com

Michel Lemieux est diplômé en production de l'École Nationale de Théâtre du Canada. Il est à la fois concepteur, scénographe, metteur en scène, compositeur, performeur et vidéaste au 4D Art. Il a également écrit, réalisé ou mis en scène plusieurs courts métrages, vidéoclips, publicités et émissions télévisuelles.

E-mail: info@4dart.com

Marta Isaacsson est professeure du Département de Théâtre et du Programme de 3 ème cycle en Arts de la Scène de l'Universidade Federal do Rio Grande do Sul, Brésil. Docteure en Études Théâtrales par l'Université de Paris III (1991). Chercheuse attachée au Conselho Nacional de Pesquisa Científica / Ministério da Ciencia e Tecnologia du Brésil.

E-mail: missilva@portoweb.com.br

Texte original et inédit en français, révisé par Annelyse Gayraud. Une traduction en portugais est publiée dans ce même numéro de la revue.

Reçu le 27 septembre 2015

Acepté le 29 décembre 2015 